\title{
Association of de novo Dipstick Albuminuria with Severe Acute Kidney Injury in Critically III Septic Patients
}

\author{
Javier A. Neyra ${ }^{a}$ John Manlloc Xilong Li $^{b}$ Gordon Jacobsen ${ }^{d}$ Jerry Yee \\ Lenar Yessayan $^{\mathrm{e}}$ f for the AKICI Study Group \\ ${ }^{a}$ Division of Nephrology, Department of Internal Medicine and bivision of Biostatistics, Department of Clinical \\ Sciences, University of Texas Southwestern Medical Center, Dallas, Tex., 'Division of Nephrology, Johns Hopkins \\ University School of Medicine, Baltimore, Md., and d Department of Public Health Sciences, and Divisions of \\ eNephrology and Hypertension and f Pulmonary and Critical Care Medicine, Henry Ford Hospital, Detroit, Mich., USA
}

\section{Key Words}

Acute kidney injury · Albuminuria · Sepsis · Dipstick

\begin{abstract}
Background: Acute kidney injury (AKI) occurs frequently in septic patients. Albuminuria may play a role as an early marker of septic AKI. The potential association between de novo dipstick albuminuria (DA) and septic AKI has not been examined. Methods: We conducted a single-center observational cohort study of 423 critically ill septic patients without chronic kidney disease (CKD) or prior positive DA within 3 months before admission. The association between de novo DA within the first $24 \mathrm{~h}$ of presentation and AKI at $72 \mathrm{~h}$ was examined. Results: AKI was identified in 268/423 (63\%) patients and 20/423 (4.7\%) required dialysis. De novo DA was associated with AKI (univariate OR 1.91; 95\% Cl 1.27-2.86, $p=0.002$ ). The association persisted in a multivariate logistic regression model adjusted for demographics, baseline kidney function, comorbidities, critical illness parameters, and exposure to nephrotoxins (adjusted OR 1.87; 95\% Cl 1.21$2.89, p=0.005)$. The association between de novo DA and AKI was stronger for severe AKI, i.e. Acute Kidney Injury Network (AKIN) stage 3 (adjusted OR 2.99; 95\% Cl 1.52-5.85, $\mathrm{p}=0.001$ ) and AKIN stage 2 (adjusted OR 1.79; 95\% Cl 1.002-3.21, p =
\end{abstract}

0.049 ) but not AKIN stage 1 (adjusted OR $1.41 ; 95 \% \mathrm{Cl} 0.87-$ $2.29, p=0.16)$. Conclusions: De novo DA within the first $24 \mathrm{~h}$ of admission was independently associated with severe AKI in critically ill septic patients. Future studies are required to fully elucidate the utility of DA testing in the early detection and stratification of AKI.

(c) 2015 S. Karger AG, Basel

\section{Introduction}

Sepsis is one of the most common reasons for intensive care unit (ICU) admissions. It often leads to multiorgan dysfunction and the kidney is one of the organs frequently affected $[1,2]$. Acute kidney injury (AKI) occurs in about $25 \%$ of patients with severe sepsis and in nearly $50 \%$ of those with septic shock [3].

Sepsis is marked by the release of a plethora of proinflammatory molecules into the systemic circulation that leads to a loss of barrier integrity of endothelial cells and systemic capillary leak $[4,5]$. The glomerular manifestation of this enhanced capillary permeability is increased excretion of albumin in the urine [6]. In experimental septic AKI, lipopolysaccharide ingestion has been shown to induce significant ultrastructural alterations in the glo-

\section{KARGER}

E-Mail karger@karger.com

www.karger.com/nec
(C) 2015 S. Karger AG, Basel

$1660-2110 / 15 / 1284-0373 \$ 39.50 / 0$
Javier A. Neyra, MD

Division of Nephrology, University of Texas Southwestern Medical Center 5323 Harry Hines Blvd.

Dallas, TX 75390-8516 (USA)

E-Mail Javier.NeyraLozano@UTSouthwestern.edu 
Fig. 1. Cohort derivation. UTI = Urinary tract infection.

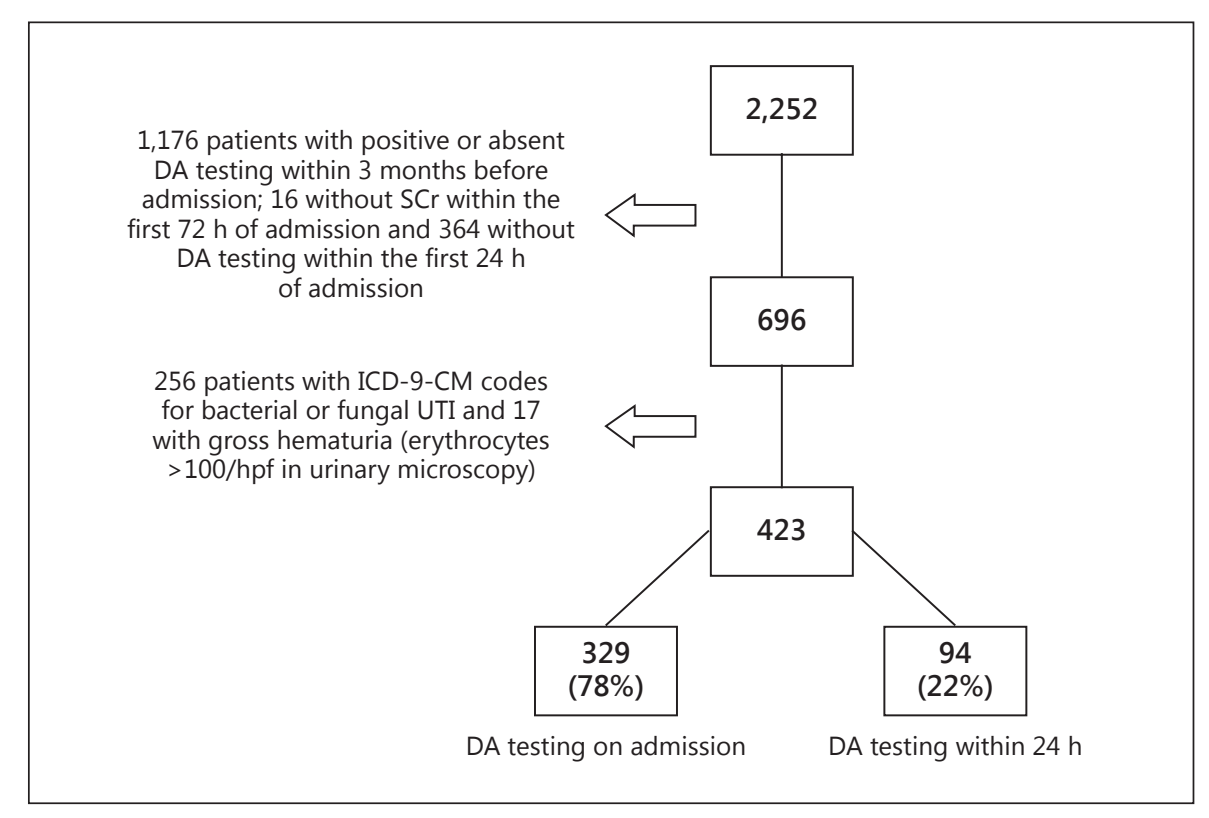

merular endothelium, and albuminuria has been detected within the first $24 \mathrm{~h}$ [7]. Furthermore, the renal cortical albumin gene, usually silent under normal conditions, has been found to be upregulated in early AKI, contributing to tubular albuminuria [8]. Thus, albuminuria may play a role as a useful marker for septic AKI.

Microalbuminuria occurs in up to $87 \%$ of septic patients [9]. Its presence in sepsis has been shown to predict vasopressor requirement, organ failure, and ICU survival [10-12]. Microalbuminuria, defined as 30-300 mg/day of albumin excretion in the urine, falls below the threshold detection of conventional urinary dipsticks. However, there may be a heightened renal excretion of albumin in septic AKI that may be detected with conventional dipsticks. Accordingly, the purpose of this study was to examine the potential association between de novo dipstick albuminuria (DA) within the first $24 \mathrm{~h}$ of ICU admission and $\mathrm{AKI}$ at $72 \mathrm{~h}$.

\section{Subjects and Methods}

\section{Study Design and Participants}

We conducted a retrospective, observational cohort study utilizing a population-based, ICU database of septic patients admitted to Henry Ford Hospital, an urban, tertiary care hospital in Detroit, Mich., USA, from January 2004 through July 2011. The subject search was done based on Angus criteria [1] for severe sepsis or septic shock using the International Classification of Diseases, Ninth Revision, Clinical Modification (ICD-9-CM) codes [13] for both a bacterial or fungal infection and a diagnosis of acute organ dysfunction excluding gastrointestinal failure. Inclusion criteria comprised adult patients admitted to the ICU with a diagnosis of severe sepsis or septic shock, a documented serum creatinine (SCr) and urinalysis within 3 months before admission, urinalysis within the first $24 \mathrm{~h}$ of admission, and at least one value of SCr within the first $72 \mathrm{~h}$ of ICU admission. Exclusion criteria consisted of preexisting chronic kidney disease (CKD; baseline SCr $>132.6 \mu \mathrm{mol} / 1$ or $>1.5 \mathrm{mg} / \mathrm{dl}$ within 3 months before admission), albuminuria detected by dipstick within 3 months before admission, pregnan$c y$, and potential causes of false-positive albuminuria on dipstick (erythrocytes $>100 / \mathrm{hpf}$ in urinary microscopy or bacterial or fungal urinary tract infection ascertained by ICD-9-CM codes). The protocol was approved by the institutional review board.

\section{Study Variables}

The most recent SCr within the 3-month period before ICU admission was defined as the baseline SCr. The greatest SCr within $72 \mathrm{~h}$ of admission was used to determine the diagnosis of AKI, defined and graded based on Acute Kidney Injury Network (AKIN) criteria [14], which defines AKI based on SCr- and urine output-based criteria. In this study, only the SCr criterion was used given the lack of urine output data. When a patient fulfilled criteria for more than one AKIN stage within the first $72 \mathrm{~h}$ of ICU admission, the higher stage was considered for the purpose of the analysis. De novo DA was defined as new-onset dipstick positive for albuminuria within the first $24 \mathrm{~h}$ of presentation with severe sepsis or septic shock in a patient who had a documented absence of DA in the past 3 months. DA was classified as either negative or positive. A positive DA consisted of a semiquantitative result from 'trace' to ' $4+$ or $>300 \mathrm{mg} / \mathrm{dl}^{\prime}$ (AUTION ${ }^{\circledR}$ Sticks 9EB; Arkray USA, Edina, Minn., USA). All subject-specific variables were obtained from electronic medical records by data management personnel blinded to the study analysis. These included demographic data (age, gender, and race), comorbidity (diabetes, hypertension, heart failure, and anemia), baseline SCr, indicators of critical illness 
Table 1. Patient characteristics by DA status

\begin{tabular}{|c|c|c|c|}
\hline Variable & Negative DA $(\mathrm{n}=228)$ & Positive DA $(\mathrm{n}=195)$ & Comparison $\mathrm{p}$ value \\
\hline \multicolumn{4}{|l|}{ Demographics } \\
\hline Age $($ mean $\pm S D)$, years & $60.7 \pm 16.8$ & $60.9 \pm 18.1$ & $0.88^{c}$ \\
\hline Male gender & $115(50.4)$ & $97(49.7)$ & $0.89^{\mathrm{e}}$ \\
\hline African-American & $120(52.6)$ & $121(62.1)$ & $0.15^{\mathrm{e}}$ \\
\hline \multicolumn{4}{|l|}{ Chronic conditions } \\
\hline Median baseline eGFR ${ }^{\mathrm{a}}$ (IQR), $\mathrm{ml} / \mathrm{min} / 1.73 \mathrm{~m}^{2}$ & $90.0(68.6-122.0)$ & $88.9(70.2-109.6)$ & $0.84^{\mathrm{d}}$ \\
\hline Diabetes & $56(24.6)$ & $56(28.7)$ & $0.33^{\mathrm{e}}$ \\
\hline Hypertension & $102(44.7)$ & $80(41)$ & $0.44^{\mathrm{e}}$ \\
\hline Systolic heart failure & $3(1.3)$ & $3(1.5)$ & $1.00^{\mathrm{f}}$ \\
\hline Anemia $^{b}$ & $221(96.9)$ & $190(97.4)$ & $0.76^{\mathrm{e}}$ \\
\hline NSAID & $14(6.1)$ & $5(2.6)$ & $0.08^{\mathrm{e}}$ \\
\hline Aminoglycoside & $51(22.4)$ & $57(29.2)$ & $0.11^{\mathrm{e}}$ \\
\hline \multicolumn{4}{|l|}{ Critical indicators } \\
\hline Median ICU length of stay (IQR), days & $5(2-11.5)$ & $5(3-9)$ & $0.95^{\mathrm{d}}$ \\
\hline Vasopressor or inotrope & $55(24.1)$ & $68(34.9)$ & $0.02^{\mathrm{e}, *}$ \\
\hline Mechanical ventilation & $107(46.9)$ & $92(47.2)$ & $0.96^{\mathrm{e}}$ \\
\hline
\end{tabular}

Values are presented as numbers (\%) unless otherwise stated. ${ }^{*} \mathrm{p}<0.05$. NSAID $=$ Nonsteroidal anti-inflammatory drug.

${ }^{a}$ Using the 4 -variable Modification of Diet in Renal Disease (MDRD) study equation. ${ }^{\mathrm{b}}$ Hematocrit $<39 \%$ for men and $<36 \%$ for women. ${ }^{c}$ Two-sided t test. ${ }^{\mathrm{d}}$ Wilcoxon rank-sum test. ${ }^{\mathrm{e}} \chi^{2}$ test. ${ }^{\mathrm{f}}$ Fisher's exact test.

(inotrope, vasopressor, diuretic use, dialysis, mechanical ventilation, and length of ICU stay), and exposure to nephrotoxins (nonsteroidal anti-inflammatory drugs or aminoglycosides).

\section{Study Outcomes}

We tested for the presence of an independent association between de novo DA within the first $24 \mathrm{~h}$ of ICU admission and AKI at $72 \mathrm{~h}$ in this selected sample of ICU patients with severe sepsis or septic shock.

\section{Statistical Analysis}

Microsoft Excel 2010 (Microsoft, Redmond, Wash., USA) and SAS 9.3 (SAS Institute, Cary, N.C., USA) were used in data acquisition and analysis. Categorical data were reported as percentages and continuous data as means \pm SD or median (IQR) as appropriate. Between-group comparisons for categorical variables were made using either the $\chi^{2}$ test or Fisher's exact test when the expected frequencies were $<5$. For continuous variables, either a 2 -sided $t$ test or a Wilcoxon rank-sum test was conducted when data were not normally distributed. In lieu of nonGaussian distributions, baseline SCr, baseline estimated glomerular filtration rate (eGFR), and ICU length of stay (days) were natural log transformed. All of the associations between potential confounders and AKI were tested by univariate logistic regression. A multivariate logistic regression model was constructed for AKI and different stages of AKIN as the dependent variables and de novo DA (dichotomized as positive or negative) as the main independent variable. This model included all covariates that may potentially be associated with AKI. Finally, a multivariate logistic regression model that adjusts for baseline kidney function was constructed using candidate variables that had a $p$ value $<0.1$ in the univariate models. The same model was used to test the association between each of AKIN stages 1-3 and de novo DA. The 95\% CI reported for the logistic regression OR were based on the Wald estimation. $\mathrm{p}<0.05$ was considered statistically significant.

\section{Results}

We identified 2,252 adult patients admitted to the ICU with the diagnosis of severe sepsis or septic shock and documented SCr $\leq 132.6 \mu \mathrm{mol} / \mathrm{l}(1.5 \mathrm{mg} / \mathrm{dl})$ within 3 months before indexed admission during the study dates. Of these, 423 patients met all inclusion and exclusion criteria (fig. 1). The baseline characteristics of the study pa- 
Table 2. Univariate association between de novo DA within the first $24 \mathrm{~h}$ of ICU admission and AKI and adverse in-hospital outcomes

\begin{tabular}{lccc}
\hline Variable & $\begin{array}{l}\text { Negative DA } \\
(\mathrm{n}=228)\end{array}$ & $\begin{array}{l}\text { Positive DA } \\
(\mathrm{n}=195)\end{array}$ & $\begin{array}{l}\text { Comparison } \\
\text { p value }\end{array}$ \\
\hline AKI at 72 h of ICU admission & & \\
All AKI & $129(56.6)$ & $139(71.3)$ & $<0.01^{\mathrm{a}, *}$ \\
AKIN 1 & $75(43.1)$ & $65(53.7)$ & $0.07^{\mathrm{a}}$ \\
AKIN 2 & $35(26.1)$ & $37(39.8)$ & $0.03^{\mathrm{a}, *}$ \\
AKIN 3 & $19(16.1)$ & $37(39.8)$ & $<0.01^{\mathrm{a}, *}$ \\
\hline Adverse in-hospital outcomes & & \\
In-hospital mortality & $51(22.4)$ & $51(26.2)$ & $0.36^{\mathrm{a}}$ \\
In-hospital dialysis & $13(5.7)$ & $7(3.6)$ & $0.31^{\mathrm{a}}$ \\
\hline
\end{tabular}

Values are presented as numbers (\%) unless otherwise stated. $* \mathrm{p}<0.05$.

${ }^{\mathrm{a}} \chi^{2}$ test.

tients, including demographic data, comorbidities, baseline SCr, indicators of critical illness, and exposure to potential nephrotoxins, are reported in table 1. No significant differences were found among patients with positive DA versus negative DA, except for the more frequent use of vasoactive drugs (e.g. vasopressor or inotrope agents) in the positive DA group. The most common diuretic utilized was furosemide, which was administered approximately $90 \%$ of the time when a diuretic was given within the first $72 \mathrm{~h}$ of ICU admission.

Of the 423 patients studied, 268 (63\%) developed AKI within the first $72 \mathrm{~h}$ of ICU admission based on AKIN criteria. Of those who developed AKI, 140 (52\%) were at stage $1,72(27 \%)$ were at stage 2 , and $56(21 \%)$ were at stage 3 . Twenty $(4.7 \%)$ patients required dialysis during the hospital admission. Urine dipstick testing was performed either at the time of ICU admission (in $78 \%$ of the patients) or during the course of the first day of ICU admission (in $22 \%$ of the patients) (fig. 1). De novo DA within the first $24 \mathrm{~h}$ of ICU admission was found in 195 (46\%) of the patients. AKI occurred more often in patients with de novo DA (table 2; fig. 2a). The semiquantitative severity categories of DA readings for each AKIN stage of AKI are reported in figure $2 \mathrm{~b}$. A total of 102 patients (24\%) died during hospital admission. The mortality rate was $28 \%$ when patients developed AKI and $17 \%$ if they did not $(\mathrm{p}=0.01)$. Of the 268 patients who developed AKI, 128 (48\%) progressed to a higher AKIN stage within the first $72 \mathrm{~h}$ of admission. De novo DA was present in 74 (58\%) but absent in $54(42 \%)$ of these patients $(\mathrm{p}=0.06)$.

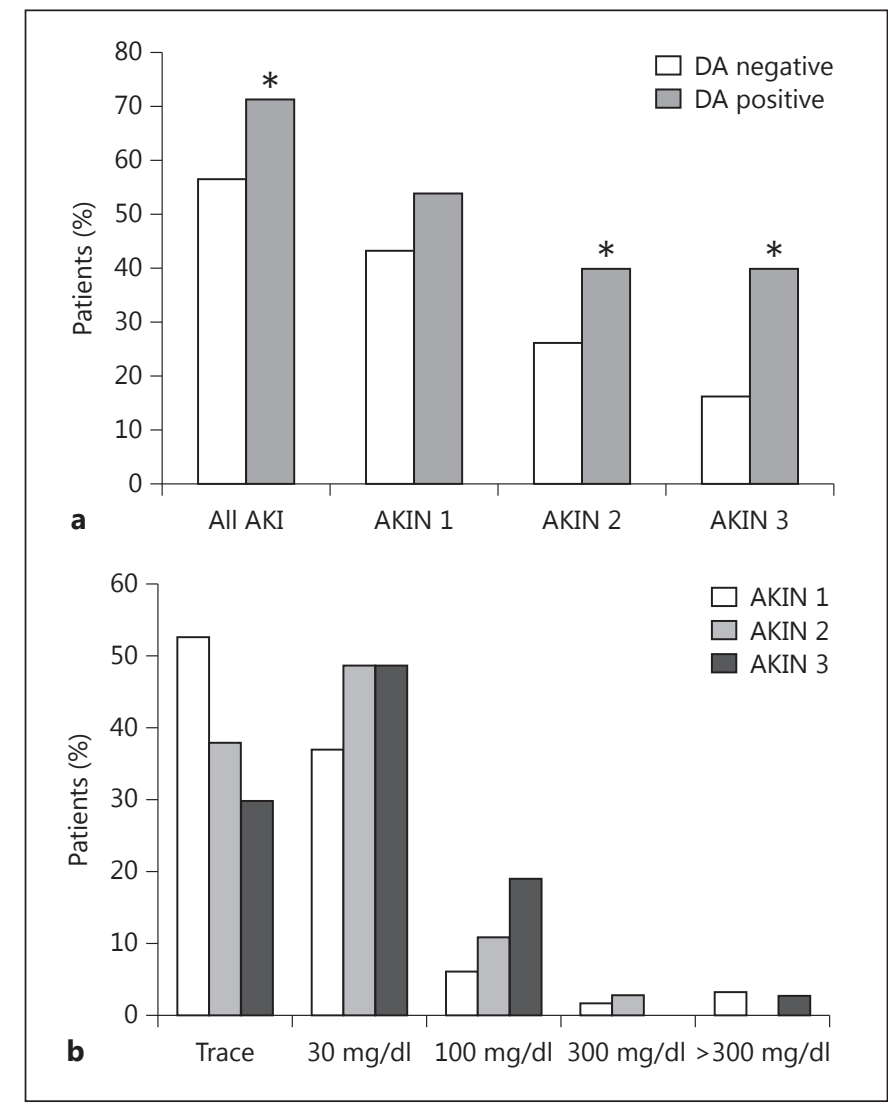

Fig. 2. a Univariate association between de novo DA within the first $24 \mathrm{~h}$ of ICU admission and AKI at $72 \mathrm{~h}$. b Semiquantitative severity categories of DA readings within the first $24 \mathrm{~h}$ of ICU admission for each AKIN stage of AKI at 72 h. ${ }^{*} \mathrm{p}<0.05$.

Univariate analysis showed a significant association between de novo DA within the first $24 \mathrm{~h}$ of ICU admission and AKI at $72 \mathrm{~h}(\mathrm{OR} 1.91 ; 95 \% \mathrm{CI} 1.27-2.86, \mathrm{p}=$ 0.002 ) (table 3 ). The association persisted in a multivariate analysis that adjusted for age, gender, race, baseline SCr, comorbidities, critical illness parameters, and exposure to nephrotoxins (adjusted OR 1.87; 95\% CI 1.21$2.89, \mathrm{p}=0.005)$ (table 3). The association between de novo DA and AKI was stronger for severe AKI, i.e. AKIN 3 (adjusted OR 2.99; 95\% CI 1.52-5.85, p = 0.001) and AKIN 2 (adjusted OR 1.79; 95\% CI 1.002-3.21, $\mathrm{p}=0.049$ ) but not AKIN 1 (adjusted OR 1.41; 95\% CI 0.87-2.29, $\mathrm{p}=0.16)($ table 4$)$. Also, the association between de novo DA and in-hospital dialysis was not statistically significant $(\mathrm{p}=0.44)$, attributable to the low number of these events $(n=20)$ in the entire cohort.

Other important predictors of AKI in our regression models were diabetes (adjusted OR 1.99; 95\% CI 1.22- 
Table 3. Association between de novo DA within the first $24 \mathrm{~h}$ of ICU admission and AKI at $72 \mathrm{~h}$

\begin{tabular}{|c|c|c|c|c|c|c|}
\hline Variable & $\begin{array}{l}\text { Univariate OR } \\
(95 \% \mathrm{CI})\end{array}$ & $\mathrm{p}$ value & $\begin{array}{l}\text { Multivariate OR } \\
(95 \% \text { CI })\end{array}$ & $\mathrm{p}$ value & $\begin{array}{l}\text { Multivariate OR } \\
(95 \% \mathrm{CI})\end{array}$ & $\mathrm{p}$ value \\
\hline De novo DA & $1.91(1.27-2.86)$ & $0.002^{*}$ & $1.87(1.21-2.89)$ & $0.005^{*}$ & $1.79(1.18-2.71)$ & $0.006^{*}$ \\
\hline Age (years) & $1.00(0.99-1.02)$ & 0.571 & $1.00(0.99-1.01)$ & 0.804 & - & - \\
\hline Male gender & $1.07(0.72-1.59)$ & 0.734 & $1.18(0.76-1.83)$ & 0.464 & - & - \\
\hline African-American vs. white & $0.67(0.32-1.42)$ & 0.359 & $0.60(0.27-1.34)$ & 0.213 & - & - \\
\hline Diabetes & $1.95(1.21-3.15)$ & $0.006^{*}$ & $2.29(1.35-3.9)$ & $0.002^{*}$ & $1.99(1.22-3.24)$ & $0.006^{*}$ \\
\hline Hypertension & $0.99(0.66-1.47)$ & 0.950 & $1.09(0.70-1.71)$ & 0.694 & - & - \\
\hline Systolic heart failure & $0.28(0.05-1.57)$ & 0.149 & $0.20(0.03-1.23)$ & 0.083 & - & - \\
\hline Anemia $^{a}$ & $3.59(1.06-12.10)$ & $0.040^{*}$ & $3.76(1.03-13.70)$ & $0.045^{*}$ & - & - \\
\hline Diuretic & $1.33(0.87-2.02)$ & 0.183 & $1.57(0.97-2.54)$ & 0.070 & - & - \\
\hline Statin & $0.71(0.44-1.16)$ & 0.170 & $0.60(0.35-1.03)$ & 0.066 & - & - \\
\hline Vasopressor or inotrope & $1.87(1.18-2.96)$ & $0.008^{*}$ & $1.61(0.97-2.68)$ & 0.065 & $1.80(1.12-2.88)$ & $0.015^{*}$ \\
\hline Mechanical ventilation & $1.33(0.89-1.98)$ & 0.162 & $1.27(0.81-2.01)$ & 0.301 & - & - \\
\hline
\end{tabular}

Data are presented in 3 models: a univariate model, a multivariate logistic regression model that adjusts for all variables, and a final model that adjusts for baseline SCr and for covariates that were retained in backward selection. For all dichotomous categorical covariates the reference is the 'nonoccurrence' of that covariate. ${ }^{*} \mathrm{p}<0.05$. NSAID $=$ Nonsteroidal anti-inflammatory drug.

${ }^{a}$ Hematocrit $<39 \%$ for men and $<36 \%$ for women.

Table 4. Multivariate logistic regression analysis adjusted for potential confounders relating de novo DA to AKIN staging

\begin{tabular}{|c|c|c|c|c|c|c|}
\hline Diabetes & $2.10(1.22-3.63)$ & $0.008^{*}$ & $1.78(0.90-3.52)$ & 0.096 & $2.15(1.00-4.64)$ & 0.051 \\
\hline Vasopressor or inotrope & $1.42(0.82-2.48)$ & 0.214 & $1.95(1.03-3.71)$ & $0.041^{*}$ & $2.86(1.44-5.70)$ & $0.003^{*}$ \\
\hline
\end{tabular}

The analysis always adjusted for baseline SCr irrespectively of statistical significance. In addition, all AKIN stages were adjusted by diabetes status and vasopressor or inotrope requirement. ${ }^{*} \mathrm{p}<0.05$.

$3.24, \mathrm{p}=0.006)$ and the use of vasoactive agents, i.e. vasopressor or inotropic drugs (adjusted OR 1.8; 95\% CI 1.12$2.88, \mathrm{p}=0.015$ ). We also performed a sensitivity analysis in which trace DA was adjudicated as negative and not positive as previously reported. We found an even stronger association between de novo DA within the first $24 \mathrm{~h}$ and AKI at $72 \mathrm{~h}$ (OR 2.11; 95\% CI 1.29-3.47, $\mathrm{p}=0.003$ ). In the multivariate model, this association remained significant (adjusted OR 1.89; 95\% CI 1.14-3.14, $\mathrm{p}=0.013$ ).

\section{Discussion}

AKI is a serious medical condition associated with high morbidity and mortality [15]. Early detection of AKI may facilitate timely intervention and mitigate kidney damage. Unfortunately, early detection of AKI is difficult and existing biomarkers of early injury have not yet been implemented in routine clinical practice [16]. Furthermore, the concept of subclinical AKI has emerged, 
that is, tubular damage without functional loss [17], and several initiatives to prevent and treat AKI are underway [18].

The presence of albuminuria, a classical risk marker of kidney disease, is becoming a red flag in the assessment of kidney risk profiles. In a population cohort, preexistent, heavy albuminuria (urine dipstick $\geq 2+$ ) predicted hospital admissions for AKI in patients with a preserved baseline kidney function [19]. Likewise, in the Atherosclerosis Risk in Communities population-based cohort, even high-normal urine albumin-to-creatinine ratios $(10-29 \mathrm{mg} / \mathrm{g})$ independently increased the risk of incident AKI in a fashion similar to decreased eGFR [20]. Moreover, in a retrospective cohort of 402 trauma patients who received intravenous contrast, the strongest predictor of contrast-induced AKI was albuminuria measured by dipstick [21]. Similarly, positive urine dipstick readings independently predicted the development of AKI in a retrospective cohort of 396 patients with severe burns [22]. Recently, Molnar et al. [23] demonstrated that early postoperative albuminuria improved the prediction of AKI in a prospective cohort of 1,198 patients undergoing cardiac surgery. DA ( $\geq 100 \mathrm{mg} / \mathrm{dl})$ was associated with the greatest risk for AKI.

Albuminuria has also shown utility in critically ill patients. In a small prospective cohort study, increasing microalbuminuria levels over the first $48 \mathrm{~h}$ predicted hospital mortality and had a high negative predictive value for the development of AKI and multiple organ failure in the ICU setting [11]. Additionally, microalbuminuria was proposed as a biomarker of systemic capillary permeability in sepsis and a useful predictor of ICU survival in comparison to common acute physiologic scores $[9,12]$.

Sepsis results from an overproduction of inflammatory mediators as a consequence of the interaction of the human immune system with pathogens and bacterial wall constituents in the body [4]. A very early feature of inflammation is endothelial dysfunction and increased capillary permeability to plasma proteins, which occurs within a few minutes of injury and is amplified by the kidney $[24,25]$. The glomerular manifestation of this enhanced capillary permeability is increased excretion of albumin in the urine [6].

Recently, animal models of AKI have been used to hypothesize the occurrence of albuminuria based on: (1) glomerular hyperfiltration, (2) glomerular injury and endothelial dysfunction, (3) tubular injury and impaired reabsorption, and (4) 'renal hepatization' or albumin gene induction. Ischemic and toxic forms of AKI can alter the glomerular structure and function, thereby en- hancing the permeability to albumin. In a mouse model of septic AKI, Kato et al. [26] identified decreases in podocin, $\mathrm{CD} 2$-associated protein (CD2AP), and tensin 2 , all essential molecules for podocyte structure and function. In that study, downregulation of these molecules was associated with foot process effacement and albuminuria after $36 \mathrm{~h}$ of injury. Additionally, Schreiber et al. [27] demonstrated that acute endotoxemia and ischemia-reperfusion-mediated AKI in mice induced downregulation of the multiligand receptors megalin and cubilin which reclaim albumin via proximal tubular endocytosis. In 2011, Ware et al. [8] described in mouse models of AKI, including ischemia-reperfusion, renal cortical expression of the normally silent albumin gene that promotes albumin secretion and thus exhibits characteristics of an acute tubular stress reactant, analogous to neutrophil-gelatinase lipocalin (NGAL) or kidney injury molecule-1 (KIM-1). This study also tested the utility of albuminuria as an early predictor of AKI, in comparison to NGAL within the first $24 \mathrm{~h}$ of injury, and determined a greater specificity in experimental AKI (murine models) and a slightly better receiver-operating characteristic curve in humans. However, the authors only studied 15 critically ill ICU patients with AKI and 14 Acute Physiology and Chronic Health Evaluation II (APACHE II)-matched controls.

Our findings highlight the strong association between new-onset or de novo DA within the first $24 \mathrm{~h}$ of ICU admission and the occurrence of severe AKI at $72 \mathrm{~h}$ in patients with severe sepsis or septic shock (e.g. negative predictive values of $92 \%$ for AKIN stage 3 and $85 \%$ for AKIN stage 2). This association was not significant for AKIN stage 1, likely because of the inability to discriminate between prerenal azotemia and intrinsic kidney injury at this mild stage of AKI. We believe that new-onset albuminuria may serve as an inexpensive biomarker in critically ill patients at risk of AKI, although it should be rigorously tested with receiver-operating characteristic analysis and performance and reclassification metrics in larger and prospective studies.

The strengths of our study include the utilization of a universally accepted definition of AKI and the use of 2 different multivariable models to adjust for confounders. Unique to our study is the exclusion of patients with advanced CKD (SCr $>132.6 \mu \mathrm{mol} / \mathrm{l}$ or $>1.5 \mathrm{mg} / \mathrm{dl}$ ) and/or prior positive DA, as well as common causes of falsepositive DA such as bacterial or fungal urinary tract infection and gross hematuria. The selection of a study sample with negative DA within 3 months prior to admission allowed us to search for the association between 
truly new-onset or de novo DA and AKI. This was not done in prior studies searching for this association. Aside from the retrospective nature of this investigation, $2 \mathrm{im}$ portant limitations are: (1) the dichotomized use of DA (positive vs. negative) where positive is both trace and 4+ or $>300 \mathrm{mg} / \mathrm{dl}$ and (2) the use of a SCr cutoff (>132.6 $\mu \mathrm{mol} / \mathrm{l}$ or $1.5 \mathrm{mg} / \mathrm{dl}$ ) and not the eGFR to exclude patients with advanced CKD, although we achieved our goal of excluding patients with CKD stages 4 and 5 (the lowest eGFR value in our study sample was $35.5 \mathrm{ml} /$ $\min / 1.73 \mathrm{~m}^{2}$ ). To address the first limitation, we performed a sensitivity analysis in which we analyzed trace DA as negative and not positive and thus confirmed our findings. This sensitivity analysis is important because a trace reading on a urine dipstick may be influenced by the urinary concentration (e.g. falsely trace $=$ truly negative in highly concentrated urine) and we did not adjust for urinary specific gravity or oliguria in our multivariate models.

In summary, this study confirms the independent association between de novo DA and severe AKI in critically ill septic patients without advanced CKD. Our study was not designed to evaluate the biomarker candidacy of de novo DA but serves to aid the design of future research in which albuminuria is quantitatively or semiquantitatively measured and its prognostic value ascertained in the presence or absence of subclinical (preserved kidney function but positive damage biomarkers) and clinically apparent AKI. Key clinical outcomes such as mortality, the need for dialysis, a worsen- ing $\mathrm{AKIN}$ stage, incident $\mathrm{CKD}$, or progressive $\mathrm{CKD}$ after AKI need to be tested.

In conclusion, de novo DA within the first $24 \mathrm{~h}$ of ICU admission was independently associated with severe AKI in critically ill septic patients. Future, prospective studies are required to test the utility of this widely available test for the early detection of AKI and to determine its predictive potential.

\section{Acknowledgements}

The authors express their gratitude to Sarah Whitehouse for expert linguistic revision of this paper and to Stephanie Stebens for expert librarian assistance formatting this paper.

The research reported in this publication was supported by the Division of Nephrology and Hypertension of Henry Ford Hospital, the University of Texas Southwestern Medical Center O'Brien Kidney Research Core Center (NIH, P30 DK079328-06), and the National Center for Advancing Translational Sciences of the National Institutes of Health (NIH, UL1TR001105). The content is solely the responsibility of the authors and does not represent the official views of the National Institutes of Health, Henry Ford Hospital, or the University of Texas Southwestern. J.A.N. is supported by the Ben J. Lipps Research Fellowship Program of American Society of Nephrology Foundation for Kidney Research and the Truelson Fellowship Fund at UT Southwestern Charles and Jane Pak Center of Mineral Metabolism and Clinical Research.

\section{Disclosure Statement}

None.

\section{References}

DA and Septic AKI
1 Angus DC, Linde-Zwirble WT, Lidicker J, et al: Epidemiology of severe sepsis in the United States: analysis of incidence, outcome, and associated costs of care. Crit Care Med 2001; 29:1303-1310.

2 Uchino S, Kellum JA, Bellomo R, et al: Acute renal failure in critically ill patients: a multinational, multicenter study. JAMA 2005;294: 813-818.

-3 Bagshaw SM, George C, Bellomo R: Early acute kidney injury and sepsis: a multicentre evaluation. Crit Care 2008;12:R47.

4 Hotchkiss RS, Karl IE: The pathophysiology and treatment of sepsis. N Engl J Med 2003; 348:138-150.

15 Aird WC: The role of the endothelium in severe sepsis and multiple organ dysfunction syndrome. Blood 2003;101:3765-3777.

6 Gosling P: Microalbuminuria: a marker of systemic disease. Br J Hosp Med 1995; 54:285290.
7 Xu C, Chang A, Hack BK, et al: TNF-mediated damage to glomerular endothelium is an important determinant of acute kidney injury in sepsis. Kidney Int 2014;85:72-81.

8 Ware LB, Johnson AC, Zager RA: Renal cortical albumin gene induction and urinary albumin excretion in response to acute kidney injury. Am J Physiol Renal Physiol 2011; 300:F628-F638.

-9 Basu S, Bhattacharya M, Chatterjee TK, et al: Microalbuminuria: a novel biomarker of sepsis. Indian J Crit Care Med 2010;14:2228.

10 MacKinnon KL, Molnar Z, Lowe D, et al: Use of microalbuminuria as a predictor of outcome in critically ill patients. $\mathrm{Br}$ Anaesth 2000;84:239-241.

11 Abid O, Sun Q, Sugimoto K, et al: Predictive value of microalbuminuria in medical ICU patients: results of a pilot study. Chest 2001; 120:1984-1988. 
12 Gosling P, Brudney S, McGrath L, et al: Mortality prediction at admission to intensive care: a comparison of microalbuminuria with acute physiology scores after 24 hours. Crit Care Med 2003;31:98-103.

13 Waikar SS, Wald R, Chertow GM, et al: Validity of International Classification of Diseases, Ninth Revision, Clinical Modification Codes for Acute Renal Failure. J Am Soc Nephrol 2006;17:1688-1694.

14 Kellum JA, Lameire N: Diagnosis, evaluation, and management of acute kidney injury: a KDIGO summary. Part 1. Crit Care 2013;17: 204.

15 Waikar SS, Liu KD, Chertow GM: Diagnosis, epidemiology and outcomes of acute kidney injury. Clin J Am Soc Nephrol 2008;3:844861.

16 McCullough PA, Shaw AD, Haase M, et al: Diagnosis of acute kidney injury using functional and injury biomarkers: workgroup statements from the tenth Acute Dialysis Quality Initiative Consensus Conference. Contrib Nephrol 2013;182:13-29.
17 Haase M, Kellum JA, Ronco C: Subclinical AKI - an emerging syndrome with important consequences. Nat Rev Nephrol 2012;8:735739.

18 Bonventre JV, Basile D, Liu KD, et al: AKI: a path forward. Clin J Am Soc Nephrol 2013;8: 1606-1608.

19 James MT, Hemmelgarn BR, Wiebe N, et al: Glomerular filtration rate, proteinuria, and the incidence and consequences of acute kidney injury: a cohort study. Lancet 2010;376: 2096-2103.

20 Grams ME, Astor BC, Bash LD, et al: Albuminuria and estimated glomerular filtration rate independently associate with acute kidney injury. J Am Soc Nephrol 2010;21:17571764.

21 Clark JJ, Wong LL, Lurie F, et al: Proteinuria as a predictor of renal dysfunction in trauma patients receiving intravenous contrast. Am Surg 2011;77:1194-1200

22 Hu JY, Meng XC, Han J, et al: Relation between proteinuria and acute kidney injury in patients with severe burns. Crit Care 2012; 16:R172.
23 Molnar AO, Parikh CR, Sint K, et al: Association of postoperative proteinuria with AKI after cardiac surgery among patients at high risk. Clin J Am Soc Nephrol 2012;7:17491760.

24 Fleck A, Raines G, Hawker F, et al: Increased vascular permeability: a major cause of hypoalbuminaemia in disease and injury. Lancet 1985;1:781-784.

25 Jensen JS, Borch-Johnsen K, Jensen G, et al: Microalbuminuria reflects a generalized transvascular albumin leakiness in clinically healthy subjects. Clin Sci (Lond) 1995;88: 629-633.

26 Kato T, Mizuno-Horikawa Y, Mizuno S: Decreases in podocin, CD2-associated protein (CD2AP) and tensin2 may be involved in albuminuria during septic acute renal failure. J Vet Med Sci 2011;73:1579-1584.

27 Schreiber A, Theilig F, Schweda F, et al: Acute endotoxemia in mice induces downregulation of megalin and cubilin in the kidney. Kidney Int 2012;82:53-59. 